\title{
Introduction to the special issue on reviews of gastric cancer metastasis and treatment
}

\author{
Masayuki Watanabe \\ 135-8550, Japan. \\ Japanese Foundation for Cancer Research, Tokyo 135-8550, Japan. E-mail: masayuki.watanabe@jfcr.or.jp \\ Cancer Metastasis Treat2018;4:48. http://dx.doi.org/10.20517/2394-4722.2018.60 \\ Received: 27 Aug 2018 Accepted: 27 Aug 2018 Published: 17 Sep 2018 \\ Science Editor: Masayuki Watanabe Copy Editor: Yuan-Li Wang Production Editor: Zhong-Yu Guo
}

Department of Gastroenterological Surgery, the Cancer Institute Hospital of Japanese Foundation for Cancer Research, Tokyo

Correspondence to: Dr. Masayuki Watanabe, Department of Gastroenterological Surgery, the Cancer Institute Hospital of

How to cite this article: Watanabe M. Introduction to the special issue on reviews of gastric cancer metastasis and treatment. $J$

It is a great honor for me to introduce the special issue entitled reviews of Gastric Cancer Metastasis and Treatment. I've been focusing on 6 topics including tumor microenvironment (TME), biomarker research, regional variation in gastric cancer treatment, diagnosis and treatment for peritoneal carcinomatosis (PC), surgical treatment for advanced or metastatic gastric cancer, and novel treatment modalities. This special issue includes 18 review articles concerning these topics.

TME has been proven to be deeply implicated in tumor progression and metastasis in gastric cancer. Sawayama et al. ${ }^{[1]}$ gave a comprehensive overview of the functions of each component of TME and reviewed the clinical impact of the alteration of TME. Cancer stem cells (CSCs) are known to be the main reason for resistance to anticancer agents as well as for the development of distant metastasis. Uchihara et al. ${ }^{[2]}$ reviewed the impact of the TME on gastric CSCs.

Biomarkers play an increasingly important role in the clinical management of cancer patients. Nakamura et al. ${ }^{[3]}$ reviewed recent progress in technology for specific enrichment and detection of circulating tumor cells (CTCs) that contribute to the diagnosis and treatment of gastric cancer. Liquid biopsy using CTCs and cell-free nucleic acids are considered as a tool that enables individualized or precision medicine. MicroRNAs (miRNAs) are short noncoding RNAs that post-transcriptionally regulate gene expression. Komatsu et al. ${ }^{[4]}$ reviewed the recent biological and clinical research on the circulating miRNAs of gastric cancer and discussed the future perspectives.

There are regional differences in recommended treatment for gastric cancer. Kamiya et al. ${ }^{[5]}$, Karolinska

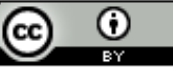

(C) The Author(s) 2018. Open Access This article is licensed under a Creative Commons Attribution 4.0 International License (https://creativecommons.org/licenses/by/4.0/), which permits unrestricted use, sharing, adaptation, distribution and reproduction in any medium or format, for any purpose, even commercially, as long as you give appropriate credit to the original author(s) and the source, provide a link to the Creative Commons license, and indicate if changes were made.

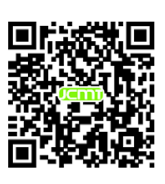


University in Sweden, reviewed current trend in gastric cancer treatment in Europe. In Europe, perioperative chemotherapy is the standard care for locally advanced gastric cancer. The regimen for the perioperative chemotherapy has shift from the Medical Research Council Adjuvant Gastric Infusional Chemotherapy (MAGIC) regimen (Epirubicin, cisplatin, 5-fluorouracil/Epirubicin, Cisplatin, Capecitabine) to the fluorouracil, folinic acid, oxaliplatin, taxotere (FLOT) triplet. Harada et al. ${ }^{[6]}$, University of Texas, M.D., Anderson Cancer Center, summarized recent trend in gastric cancer in the USA. In the USA, postoperative chemoradiation is one of the standard care for locally advanced tumors. When cancer progresses after the first line therapy, additional biomarkers, including microsatellite instability (MSI) and programmed deathligand 1 (PD-L1) should be tested for the screening of candidates for the checkpoint inhibitors. Eto et al. ${ }^{[7]}$, Cancer Institute Hospital in Japan, reviewed recent publications and guidelines focusing on the progress in treatment of metastatic gastric cancer in Japan. The incidence of adenocarcinoma in the esophagogastric junction (EGJ) has been increasing rapidly, especially in Western countries. Although treatment for EGJ adenocarcinoma has been developed as a type of gastric cancer, recent comprehensive molecular analysis revealed differences in molecular mechanisms between EGJ and gastric adenocarcinomas. Toihata et al. ${ }^{[8]}$ reviewed recent evidence of treatment for advanced EGJ adenocarcinoma.

PC is frequently observed in patients with advanced gastric cancer and is considered to be an incurable disease. Hu et al..$^{[9]}$ reviewed the molecular mechanisms of three steps in the development of PC, including detachment from the primary tumor, adaptation to the microenvironment of the peritoneal cavity, and attachment to peritoneal mesothelial cells. Peritoneal lavage cytology (PLC) has been shown to be an independent predictor of cancer relapse after curative gastrectomy and poor prognosis. Matsuoka and Yashiro $^{[10]}$ reviewed the clinical roles and attributes of PLC in gastric cancer. Sugarbaker ${ }^{[11]}$ summarized the role and efficacy of neoadjuvant systemic chemotherapy, neoadjuvant intraperitoneal and systemic chemotherapy, cytoreductive surgery, and perioperative chemotherapy including hyperthermic intraperitoneal chemotherapy and/or early postoperative intraperitoneal chemotherapy as prevention or treatment for PC. Macedo et al. ${ }^{[12]}$ introduced pressurized intraperitoneal aerosol chemotherapy as a treatment option for PC.

Pergolini et al. ${ }^{[13]}$ performed a systematic review of literature on surgical resection for metastatic gastric cancer. Survival benefit of surgery in advanced gastric cancer is still unclear. Surgery may play an important role in highly selected patients. However, further randomized controlled trials are necessary to clarify the actual impact of surgery in these patients. Recent advances in chemotherapy enabled conversion surgery for patients with initially unresectable gastric cancer. Ida and Watanabe ${ }^{[14]}$ reviewed the treatment strategies for stage IV gastric cancer and discussed the potential efficacy of conversion surgery. Pancreaticoduodenectomy (PD) is the only possible treatment for achieving Ro resection when a tumor and/ or lymph node metastasis directly invades the pancreatic head or infiltrates the duodenum. However, the efficacy and safety of PD for advanced gastric cancer remain unclear. Makuuchi et al ${ }^{[15]}$ reviewed the literatures on PD for gastric cancer and their own experience.

Recently, targeting therapies and immune checkpoint blockade have been introduced into gastric cancer treatment. Kiyozumi et al. ${ }^{[16]}$ summarized the latest knowledge of focused common cancer targets, signaling pathways, targeting therapies, and immunotherapies for gastric cancer. The late-phase complication of the large-extent of gastric resection negatively influences patients' quality of life. Takeuchi and Kitaga$\mathrm{wa}^{[17]}$ introduced current status of sentinel lymph node (SN) biopsy and function-preserving gastrectomy based on the SN biopsy. Robotic assisted surgery is increasingly performed for many types of cancers. Tokunaga et al ${ }^{[18]}$ reviewed the comparative retrospective and prospective studies which have investigated the difference in short- and long-term outcomes between robotic gastrectomy and laparoscopic gastrectomy.

I would like to express my sincere gratitude to Professor Lucio Miele, Editor-in-Chief, Journal of Cancer Metastasis and Treatment, for giving me this opportunity. I would like to thank all of the contributing au- 
thors for their hard work in producing the articles. I also thank Professor Hideo Baba, Department of Gastroenterological Surgery, Kumamoto University, for his guidance and support for this project. I am very happy if you enjoyed this special issue.

\section{DECLARATIONS}

\section{Authors' contributions}

The author contributed solely to the article.

\section{Availability of data and materials}

Not applicable.

\section{Financial support and sponsorship}

None.

\section{Conflicts of interest}

The author declared that there are no conflicts of interest.

\section{Ethical approval and consent to participate}

Not applicable.

\section{Consent for publication}

Not applicable.

\section{Copyright}

(C) The Author(s) 2018.

\section{REFERENCES}

1. Sawayama H, Ishimoto T, Baba H. Microenvironment in the pathogenesis of gastric cancer metastasis. J Cancer Metastasis Treat 2018;4:10.

2. Uchihara T, Ishimoto T, Yonemura A, Baba H. Therapeutic targets against gastric cancer stem cells interacting with tumor environment. $\mathrm{J}$ Cancer Metastasis Treat 2018;4:9.

3. Nakamura K, Iwatsuki M, Kurashige J, Ishimoto T, Baba Y, Miyamoto Y, Yoshida N, Watanabe M, Baba H. Circulating tumor cells in gastric cancer. J Cancer Metastasis Treat 2018;4:32.

4. Komatsu S, Kiuchi J, Imamura T, Ichikawa D, Otsuji E. Circulating microRNAs as a liquid biopsy: a next-generation clinical biomarker for diagnosis of gastric cancer. J Cancer Metastasis Treat 2018;4:36.

5. Kamiya S, Rouvelas I, Lindblad M, Nilsson M. Current trends in gastric cancer treatment in Europe. J Cancer Metastasis Treat 2018;4:35.

6. Harada K, Baba H, Ajani JA. Recent trend in gastric cancer treatment in the USA. J Cancer Metastasis Treat 2018;4:18.

7. Eto K, Ida S, Watanabe M, Baba H. Treatment strategy for metastatic gastric cancer in Japan. J Cancer Metastasis Treat 2018;4:23.

8. Toihata T, Imamura Y, Watanabe M, Baba H. Management of metastatic esophagogastric junction adenocarcinoma. J Cancer Metastasis Treat 2018;4:24.

9. Hu QJ, Ito S, Yanagihara K, Mimori K. Molecular mechanism of peritoneal dissemination in gastric cancer. J Cancer Metastasis Treat 2018;4:39.

10. Matsuoka T, Yashiro M. Significance of peritoneal lavage cytology based on genetic signatures in gastric cancer. J Cancer Metastasis Treat 2018;4:6.

11. Sugarbaker PH. Gastric cancer: prevention and treatment of peritoneal metastases. J Cancer Metastasis Treat 2018;4:7.

12. Macedo F, Ladeira K, Longatto-Filho A, Martins SF. Gastric cancer treated with pressurized intraperitoneal aerosol chemotherapy: revising an option for peritoneal carcinomatosis. J Cancer Metastasis Treat 2018;4:8.

13. Pergolini I, Ciano P, Guercioni G, Catarci M. Surgical treatment of stage IV gastric cancer: is it worthwhile? J Cancer Metastasis Treat 2018;4:33.

14. Ida S, Watanabe M. Conversion surgery for stage IV gastric cancer. J Cancer Metastasis Treat 2018;4:22.

15. Makuuchi R, Irino T, Tanizawa Y, Bando E, Kawamura T, Terashima M. Pancreaticoduodenectomy for gastric cancer. J Cancer Metastasis Treat 2018;4:26.

16. Kiyozumi Y, Iwatsuki M, Yamashita K, Koga Y, Yoshida N, Baba H. Update on targeted therapy and immune therapy for gastric cancer, 2018. J Cancer Metastasis Treat 2018;4:31. 
17. Takeuchi H, Kitagawa Y. Laparoscopic personalized function-preserving gastrectomy with sentinel node mapping for early-stage gastric cancer. J Cancer Metastasis Treat 2018;4:38

18. Tokunaga M, Watanabe M, Sugita S, Tonouchi A, Kaito A, Kinoshita T. Robotic gastrectomy for gastric cancer. J Cancer Metastasis Treat 2018;4:40. 